\title{
Serpiginous choroiditis: an unusual presentation of ocular sarcoidosis
}

\author{
C Edelsten, M R Stanford, E M Graham
}

Choroiditis is frequently found in patients with posterior segment inflammation associated with sarcoidosis. The most frequent pattern of choroidal involvement is areas of well circumscribed pre-equatorial choroidal depigmentation that become associated with retinal pigment epithelial (RPE) hyper- or hypopigmentation with time. ${ }^{1-4}$ We present two patients with biopsy proved sarcoidosis in whom there was extensive, confluent choroiditis with RPE changes resembling serpiginous choroiditis.

\section{Case reports}

CASE 1

A white woman developed intermittent bilateral anterior uveitis at the age of 23 that required topical steroids, and occasionally systemic prednisolone, to control. At 33 she developed an optic neuropathy that resolved spontaneously. Despite the absence of any systemic symptoms a Kveim test was positive. Examination at age 44 showed visual acuity in the right eye of $6 / 4$ and in the left eye of $6 / 18$ with a left relative afferent pupillary defect. She had left optic atrophy and the right fundus showed extensive choroidal lesions (Fig 1A) whose edges masked early

Medical Eye Unit, St Thomas's Hospital, London SE1 7EH C Edelsten

M R Stanford

E M Graham

Correspondence to: DrE M Graham.

Accepted for publication 26 August 1993

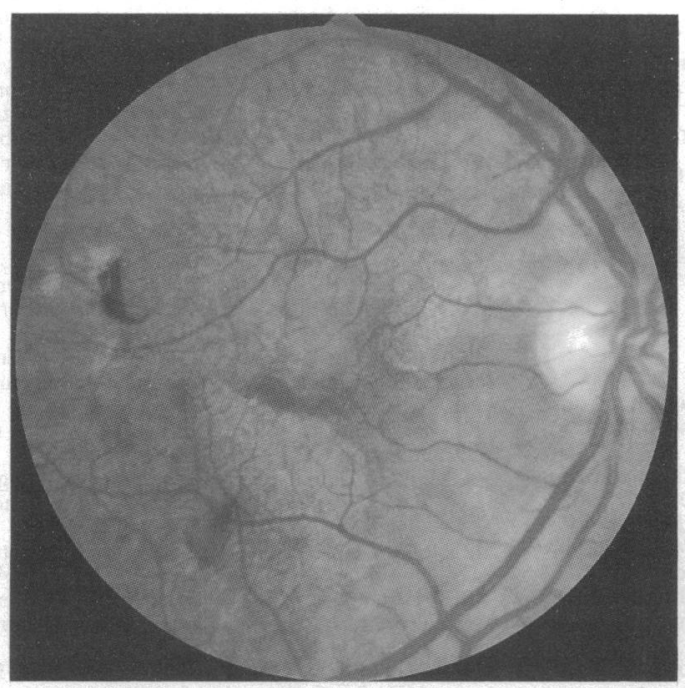

\section{Fig $1 A$}

Figure 1 (A) Fundus photograph (case 1) showing extensive areas of pigment epithelial atrophy and some clumping. $(B)$ Early and $(C)$ late fluorescein angiographic pictures in case 1 showing patchy early filling and late staining in a serpiginous pattern.

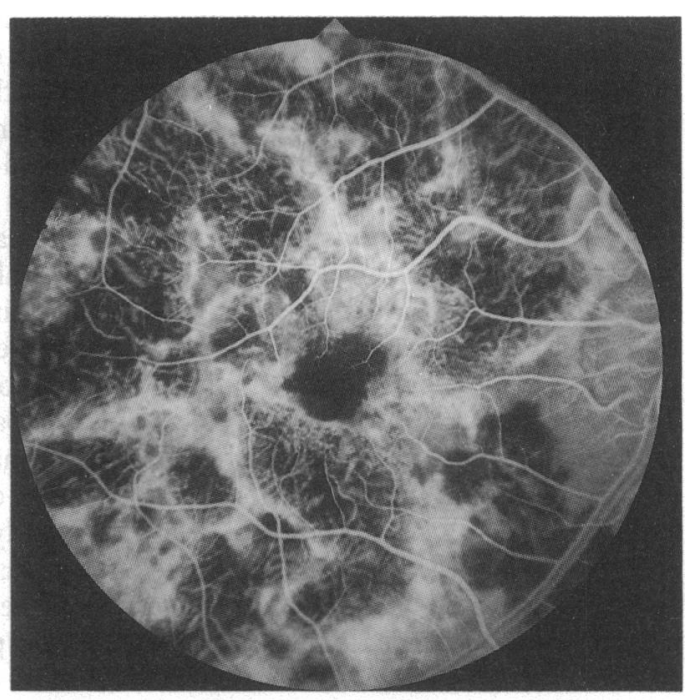

Fig $1 B$

and leaked late on fluorescein angiography (Fig 1B, 1C).

\section{CASE 2}

A white woman developed bilateral choroiditis at age 29 and was treated with antituberculosis therapy, despite there being no extraocular evidence of tuberculosis. At age 47 she developed an enlarged cervical lymph node which on biopsy showed the typical changes of

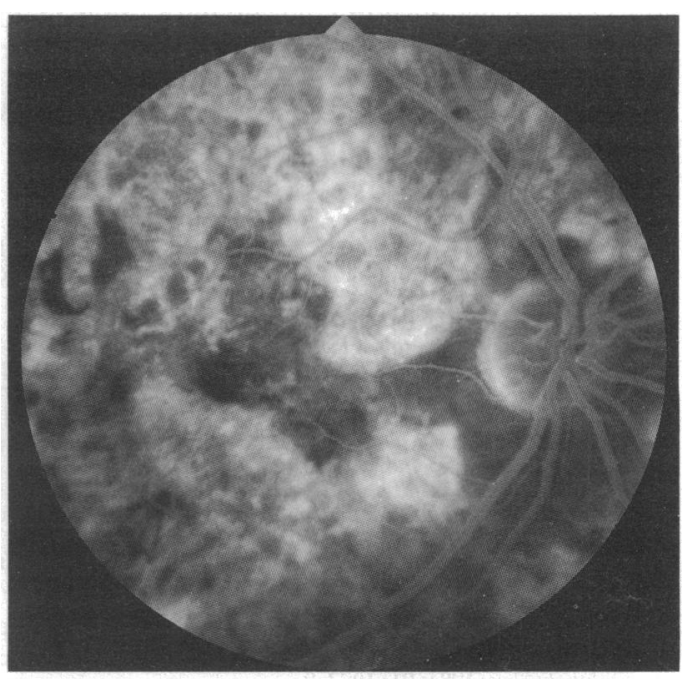


Figure 2 (A) Fundus photograph (case 2) showing marked pigment epithelial atrophy with clumping.

Fluorescein angiography shows marked atrophy in the early film $(B)$ and late staining at the edge of the lesion in the late pictures $(C)$.

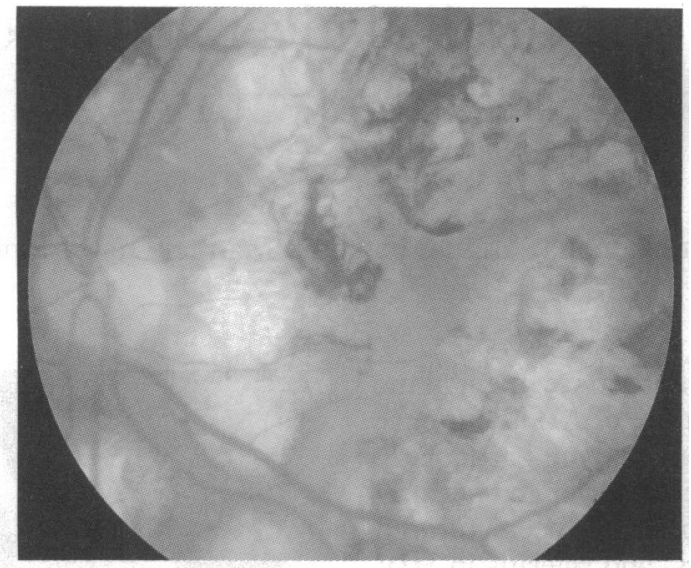

Fig $2 A$

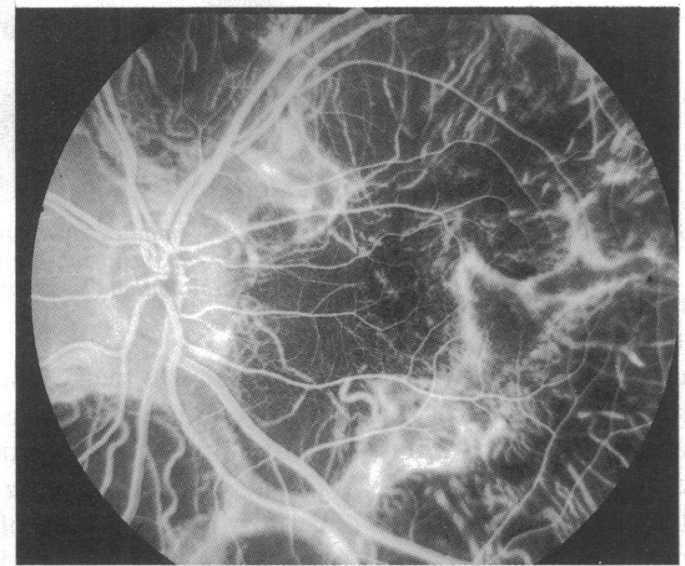

Fig $2 B$

sarcoidosis. Her vision at that time was $6 / 24$ right eye, 6/6 left eye. At age 70 she developed a reactivation of her disease in the right eye and the vision dropped to counting fingers despite prolonged treatment with oral prednisolone. At that time both fundi showed extensive choroidal lesions (Fig 2A) whose edges masked early and leaked late on fluorescein angiography (Fig 2B, 2C).

\section{Comment}

Posterior uveitis occurs in $12 \%$ of all cases of sarcoidosis and is more common in whites and females. ${ }^{4}$ Choroidal involvement may be seen in $29-50 \% .^{23}$ These lesions are most frequently seen in the inferior peripheral fundus, and it is unusual for them to occur at the posterior pole. They usually appear as patches of creamy yellow choroidal depigmentation, which are later associated with overlying hypo- or hyperpigmentation of the RPE. The clinical appearance of these lesions is non-specific and aggressive investigation of patients with multifocal choroiditis and panuveitis may show localised sarcoidosis. There has only been one previous report of extensive changes accompanying a diffuse widespread

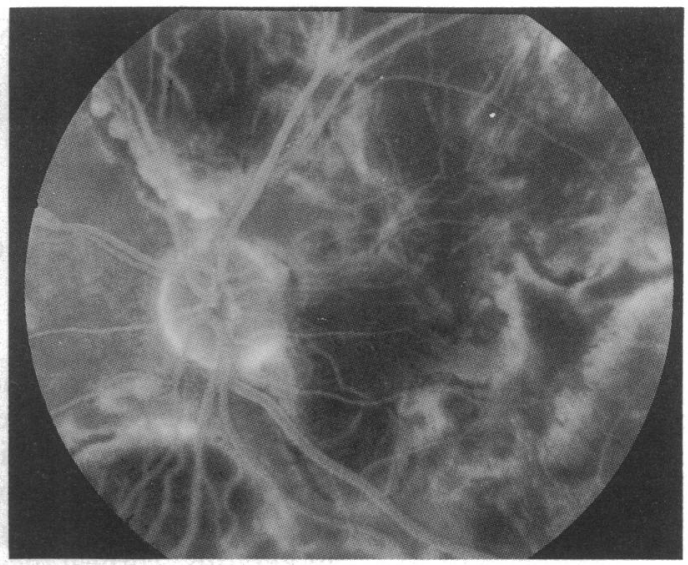

Fig 2C

choroiditis in sarcoidosis, but this was never observed in its active stages. ${ }^{5}$

Both of our patients demonstrated extensive areas of posterior pole choroiditis associated with marked RPE changes with the fluorescein angiographic features of early masking and late staining of the edge of the lesions. Progression of the disease, causing marked visual loss, occurred without significant retinal inflammation. Thus both patients showed a clinical resemblance to serpiginous choroiditis. Serpiginous choroiditis is a clinical entity which has been described in white people aged $20-50$, and is associated with vitritis, subretinal neovascularisation, and disc oedema. Helicoid progression from the disc is not universal, and disease starting both peripherally and from the macula has been described. No association with sarcoidosis has been reported previously, but it is of interest that the early reports of this disease noted a high incidence of active or presumed tuberculosis. ${ }^{6}$ Histological reports are rare and describe a round cell infiltrate of the choriocapillaris and part of the choroid, without granuloma formation. In summary, we report the clinical and angiographic features of two patients with confluent choroiditis resembling serpiginous choroiditis in whom there was evidence of systemic sarcoidosis.

The authors would like to thank Professor W I MacDonald, The authors would like to thank Professor W I MacDonald, for allowing us to report case 1 and Mrs Dora Paterson for for allowing us to report
preparing the manuscript.

1 Sanders MD, Shilling JS. Retinal, choroidal, and optic disc involvement in sarcoidosis. Trans Ophthalmol Soc UK 1976; 96: $140-4$.

2 Spalton DJ, Sanders MD. Fundus changes in histologically confirmed sarcoidosis. Br f Ophthalmol 1981; 65: 348-58.

3 Graham EM. Ocular involvement in sarcoidosis. Sem Resp Med 1986; 8: 59-64.

4 Rothova A, Alberts C, Glasius E, Kijlstra A, Buttenhuis H, Breebaart A. Risk factors for ocular sarcoidosis. Doc Ophthalmol 1989; 72: 287-96.

5 Flore PM, Friedman AH. Unusual chorioretinal degeneration associated with sarcoidosis. Am $\mathcal{f}$ Ophthalmol 1988; 106: $490-1$.

6 Laatikainen L, Erkkila H. Serpiginous choroiditis. $\mathrm{Br} f$ Ophthalmol 1974; 58: 777-83. 